\title{
Plaque morphology detected with Duplex ultrasound before carotid angioplasty and stenting (CAS) is not a predictor of carotid artery in-stent restenosis, a case control study
}

\author{
Katrin Wasser ${ }^{1}$, André Karch¹, Sonja Gröschel², Janin Witzenhausen', Klaus Gröschel, Mathias Bähr'
} and Jan Liman ${ }^{1 *}$

\begin{abstract}
Background: In-stent restenosis (ISR) is an important factor endangering the long-term safety and efficacy of carotid artery angioplasty and stenting (CAS). It is plausible that soft vulnerable plaques are more likely to be injured during CAS procedure and are therefore more likely to initiate the cascade finally leading to ISR. The aim of this study was to investigate if plaque morphology detected by a simple applicable Duplex ultrasound score before CAS can be used as a predictor for ISR.

Methods: Within a prospectively collected single-centre CAS database of 281 patients (comprising 300 arteries) with high-grade carotid artery stenosis, who underwent CAS between May 2003 and January 2013, we conducted a nested case-control study. Plaque morphology before CAS was analysed by a blinded investigator and each parameter of the Total Plaque Risk Score (TPRS) as well as the whole score was evaluated with regard to its diagnostic validity for ISR.

Results: We analysed the data of 10 patients with ISR and 50 patients without ISR. There were no significant differences with respect to baseline characteristics, vascular risk factors, and degree of stenosis between patients with and without ISR. The duration of follow-up was longer in patients with ISR $(p=0.024)$ and these patients were more likely to show increased PSV ( $p=0.012)$ immediately after CAS than patients without ISR. Neither individual parameters of the TPRS score nor the score as a whole were suitable as a diagnostic test for ISR development.

Conclusions: In the present study we could demonstrate that the non-contrast enhanced DUS of the pre-interventional plaque formation cannot be used as a predictor for the development of ISR. Evaluating a more sophisticated, but not routinely available approach e.g. by ultrasound based plaque perfusion imaging or CT based plaque analysis could be helpful in the future in order to assess the role of plaque morphology in the context of ISR development.
\end{abstract}

Keywords: Carotid artery stenosis, Stent, Angioplasty, Restenosis, Plaque, Duplex ultrasound

\section{Background}

Atherosclerotic stenosis of the internal carotid artery (ICA) is known as a major risk factor for disabling stroke and death. Carotid endarterectomy (CEA) in combination with best medical treatment of concomitant cerebrovascular risk factors is currently the therapy standard for patients with symptomatic ICA stenosis and some patients with high-

\footnotetext{
*Correspondence: jliman@gwdg.de

'Department of Neurology, University of Göttingen, Robert-Koch-Str. 40, 37075 Göttingen, Germany

Full list of author information is available at the end of the article
}

grade asymptomatic ICA stenosis. Nevertheless, carotid angioplasty and stenting (CAS) has been used as the treatment of first choice in many centres, despite the fact that randomized controlled trials and subsequent meta-analyses could not provide evidence for a general superiority of CAS over CEA [1-6]. However, the results of these trials have been interpreted very controversially resulting in conflicting recommendations in various current guidelines $[7,8]$. Although CEA is still the goldstandard therapy for most patients, there is accumulating evidence that a subgroup of patients aged $<70$ years may benefit from a CAS

\section{Ciomed Central}


intervention [5,9-11]. One factor that could influence the long-term safety and efficacy of CAS is an in-stent restenosis (ISR); indeed, we could recently show that the combined stroke and death rate during long-term follow-up was significantly higher in the group of patients suffering from ISR compared with patients without ISR [12]. Therefore, it is of highest interest to identify predictors of carotid artery in-stent restenosis in CAS-treated patients.

A possible predictor of ISR could be the lesion characteristics of the stenotic artery. Up to now it could be shown that (regardless of a CAS intervention) a compound score of plaque surface irregularity, echoluency and texture characteristics can predict the risk of stroke [13]. Furthermore, new ischemic lesions as detected with MRI after CAS are closely related to the plaque vulnerability. It could also be shown that fibrolipid plaques are associated with a higher burden of new ischemic lesions [14,15].

Taking pathophysiological mechanisms of ISR development into account, it seems plausible that plaque morphology is not only a predictor of ischemic events during CAS, but may also be associated with a higher risk of the development of an ISR. We know from coronary artery angioplasty and stenting that vascular injury, which is caused by balloon inflation and stent placement, leads to inflammatory processes, which, themselves, play the pivotal role in the pathogenesis of ISR, finally causing neointimal proliferation through the stent meshes [16-18]. In the context of CAS Petric et al. demonstrated that calcified plaques bear a lower risk of arterial injury although they could be exposed to higher dilation pressure while CAS. The authors concluded that this might reduce the initial stimulus for ISR [19].

The Total Plaque Risk Score (TPRS) described by Prati et al. [13] has proven its value in the prediction of future strokes in asymptomatic patients and considers 1) the degree of the stenosis, 2) echogenicity, 3) texture, and 4) surface characteristics. We used this score, which can easily be evaluated with the commonly available, costeffective and non-invasive Duplex ultrasound investigation, in order to investigate the influence of plaque morphology on ISR development.

\section{Methods}

\section{Patients}

The design of the core study has been published in detail, recently [12]. Within a prospectively created singlecentre CAS database of 281 patients (comprising 300 arteries) we conducted a nested case-control study. All patients suffered from a symptomatic carotid artery stenosis $\geq 70 \%$ or an asymptomatic carotid artery stenosis $\geq 90 \%$ (degree of stenosis was measured according to the European guidelines (ECST) [20]) and underwent CAS between May 2003 and January 2013. For inclusion in this study, only patients with a complete and well- documented pre-interventional Duplex ultrasound were considered. A total of 14 patients (4.7\%) developed ISR during long-term follow-up within our database. Of these 14 patients, $10(71.4 \%)$ had well analysable, preinterventional Duplex ultrasound image. Furthermore, a control-group of 50 patients (23.8\%) without ISR during long-term follow-up was randomly chosen from those meeting above named inclusion criteria for cases $(\mathrm{n}=$ 210). All cardiovascular risk factors and clinical outcome parameters were recorded by experienced stroke neurologists (K.G. and K.W.).

The current study has been conducted in accordance with International Conference on Harmonisation/Good Clinical Practice (ICH/GCP) guidelines and was approved by the local Ethics committee of the University Hospital Göttingen, Germany.

\section{Doppler and duplex sonography}

The diagnosis of carotid artery stenosis and ISR was made by carotid duplex ultrasound imaging using a combination of direct and indirect criteria, which have been described in detail, recently [12,21]. Peak systolic flow velocities (PSV) within the stenosis and post-stenotic internal carotid artery, end diastolic flow velocity in the stenosis, internal carotid artery/common carotid artery PSV ratio, and pre-stenotic and post-stenotic frequency patterns were determined as direct criteria for the local degree of stenosis. Flow characteristics of the supra-trochlear artery and the anterior cerebral artery as well as the pulsatility of the ipsilateral common carotid artery were taken into account as indirect criteria for a high-grade stenosis. The degree of carotid stenosis at baseline was graded according to angle corrected maximum intrastenotic peak systolic velocities according to ECST criteria as follows: baseline stenosis $\geq 70 \%=P S V \geq 200 \mathrm{~cm} / \mathrm{s}$, baseline stenosis $\geq 80 \%=$ PSV $\geq 300 \mathrm{~cm} / \mathrm{s}$, baseline stenosis $\geq 90 \%=P S V \geq 400 \mathrm{~cm} / \mathrm{s}$.

As there is a lack of valid ultrasound criteria for the definition of an ISR and as the current literature supposes different criteria, $[5,6,22]$ we used locally adopted criteria with a PSV $\geq 300 \mathrm{~cm} / \mathrm{s}$ as a key feature representing an ISR of $\geq 70 \%$, as this velocity is best evaluated in the literature and consistent with our CT or angiography based control examinations of ISR detected by DUS [12,21].

Regarding to plaque morphology we collected data with respect to four different plaque qualities as described in detail by Prati et al. [13] (Table 1). The first parameter is the degree of stenosis. According to Prati et al., patients with a stenosis $>40 \%$ using NASCET criteria were scored with " 1 ". All of our patients fulfilled that criterion. Secondly, the echogenicity was graded from 1 to 3 according to the Gray-Weale modified score [23-25]. Thirdly, the texture was graded " 1 " if a heterogeneous echo pattern was detected and " 0 " if a homogeneous echo pattern was present. 
Table 1 The Total Plaque Risk Score (TPRS)

\begin{tabular}{lcccc}
\hline & Parameter & Score $=\mathbf{0}$ & Score $=\mathbf{1}$ & Score $=\mathbf{2}$ \\
\hline I) & Degree of stenosis & $<40 \%$ (NASCET) & $\geq 40 \%$ (NASCET) & Intermediate echogenicity \\
II) & Echogenicity & & Hyper-echogenicity \\
III) & Texture & Homogeneous & Heterogeneous & \\
IV) & Surface & Smooth & Irregular & \\
\hline
\end{tabular}

Fourthly, the surface characteristic was graded " 0 " if the plaque contour was smooth or " 1 " if it was irregular. The TPRS was computed by adding the values of the four parameters and could range from 0 to 6 as summarized in Table 1. We also composed an alternative score based on the latter three parameters with inversed values for the parameter "echogenicity", because an anechoic plaque is as well as a heterogeneous texture and an irregular surface considered being associated with a higher vulnerability of the plaque (Figure 1).

All examinations were performed according to a standardized protocol in the same vascular laboratory with the same ultrasound equipment (Acuson Sequoia ${ }^{\mathrm{ma}}$ 512, Siemens, San José, CA) under the supervision of an experienced, board certified vascular neurologist (K.G.).

Patient data sets were collected by a second person of the study group (K.W.) and subsequently analysed by an experienced, board certified vascular neurologist, blinded to outcome parameters (J.L.).

\section{CAS procedure}

CAS was carried out by experienced interventional neuroradiologists under anaesthesiological stand-by using a transfemoral approach. Stent-type and the use of filterbased neuroprotection devices were chosen at the discretion of the interventionalists. Only patients scheduled for elective CAS were recorded, patients in unstable clinical conditions or with stroke in evolution were excluded. All patients received orally administered acetylsalicylic acid (100 mg/d) and clopidogrel $(75 \mathrm{mg} / \mathrm{d})$ at least 3 days before the procedure or if that was not possible due to CAS procedure earlier than 3 days after admission they received a loading dose of $600 \mathrm{mg}$ clopidogrel and $300 \mathrm{mg}$ of acetylsalicylic acid. Clopidogrel was continued for 6 to 12 weeks after CAS in a daily dosage of $75 \mathrm{mg}$ and aspirin was administered life-long in a dosage of $100 \mathrm{mg} / \mathrm{d}$. After being routinely monitored in our intensive care or stroke unit overnight for at least one day all patients could be discharged to normal ward or home.

\section{Follow-up protocol}

All patients were seen for serial duplex sonography and clinical follow-up at the hospital's outpatient clinic at 3, 6 , and 12 months after the CAS-procedure and every 6 months thereafter.

\section{Statistical analysis}

Categorical variables were expressed as count and percentages, continuous values as mean \pm standard deviation (SD), or as median values with the corresponding interquartile range (IQR) as appropriate. For univariate comparisons of categorical data, Chi-square tests with Yates' correction and Fisher's exact test were used as appropriate.
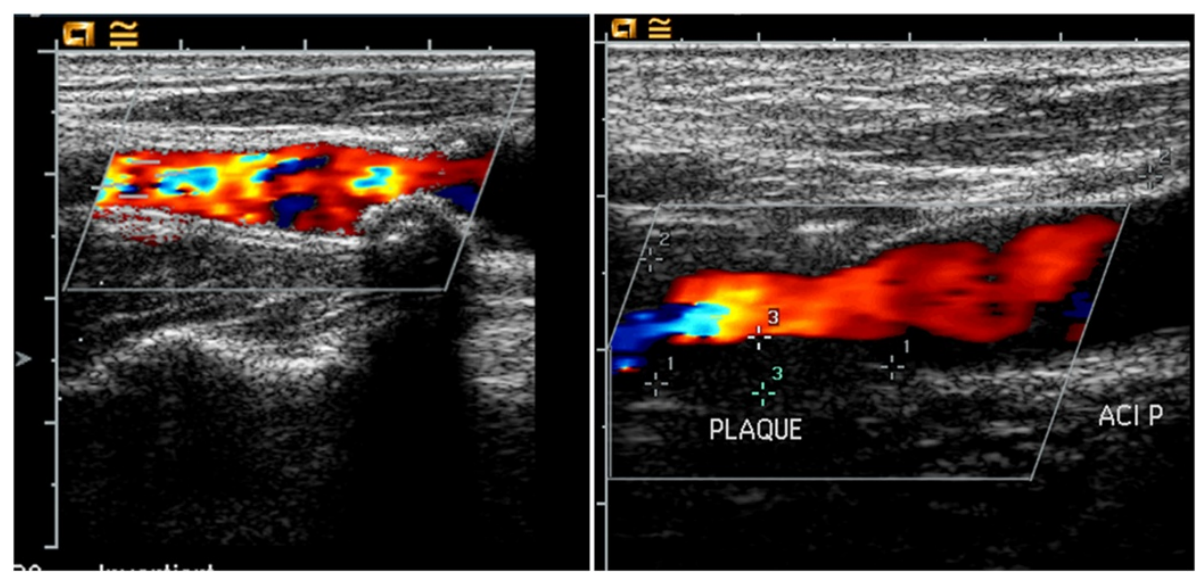

Figure 1 Shown are two plaque types with different plaque scores. The left plaque would be classified as stenosis $<40 \%$; intermediate echogenicity; heterogenous texture and irregular surface. Plaque score: 4; reversed score: 4 . The right plaque would be classified as stenosis $<40 \%$, low echogenicity, homogeneous texture and smooth surface. Plaque score: 1, reversed score: 4. 
For univariate comparisons of continuous outcome variables, two-sided t-tests and Wilcoxon-Ranksum tests were applied dependent on distribution features and variances of the outcome variable.

TPRS was evaluated as a diagnostic test by using receiver-operating characteristic (ROC) curves. Sensitivity and specificity was calculated for different cut-off values to establish the best cut-off according to the Youden-index. Predictive values were calculated with the Bayes formula using prevalence estimates from the initial cohort study.

In an exploratory secondary analysis, respective score items were assessed individually as potential diagnostic tests for predicting in-stent restenosis in patients who underwent CAS.

All statistical analyses were performed with Stata 11 (StataCorp, College Station, Texas).

\section{Results}

A total of sixty patients (48 men and 12 women) treated with CAS were analyzed in this study including 10 cases (patients with ISR) and 50 controls without ISR. Cases and controls showed no significant differences with respect to baseline characteristics, vascular risk factors, and degree of stenosis (Table 2). ISR cases were followed up for longer $(p=0.024)$ and were more likely to show increased PSV $(\mathrm{p}=0.012)$ post intervention than controls.

\section{Evaluation of plaque morphology}

When investigating plaque morphology before CAS all patients in the ISR group as well as in the control group met the criteria of stenosis degree $>40 \%$.

Echogenicity was scored as described by Prati et al. [13]. In the ISR group 50\% (5 of 10 patients) met the criteria for score $1,30 \%$ ( 3 of 10 patients) for score 2 and $20 \%$ ( 2 of 10 patients) for score 3 . The mean score in the ISR group was 1,7 . In comparison, in the control group $34 \%$ scored 1 (17 of 50 patients), 54\% scored 2 (27 of 50 patients) and $12 \%$ scored 3 (6 of 50 patients) with a mean echogenicity score of 1.78 . No significant difference was detectable between the two groups $(p=0,46)$.

As the degree of hyperechogenicity correlates with the calcification and might also be beneficial, in contrast to a "soft plaque", we also reversed the TPRS scoring system in this point scoring 1 for hyperechogenicity and 3 for low echogenicity. This sub score showed a hyperechogenicity in 2 of 10 patients (20\%) in the ISR group and in 6 of 50 patients (12\%) in the group of patients

Table 2 Baseline characteristics of study population

\begin{tabular}{|c|c|c|c|}
\hline \multirow[t]{2}{*}{ Variable } & \multicolumn{3}{|c|}{ Data } \\
\hline & ISR $\geq 70 \%$ & No ISR & $\mathrm{p}$ value \\
\hline$\overline{\mathrm{N}}$ & 10 & 50 & \\
\hline Age, years & $69.8 \pm 7.6$ & $68.2 \pm 8.7$ & 0.580 \\
\hline Female sex & $4(40 \%)$ & $8(16 \%)$ & 0.101 \\
\hline Right side & $6(60 \%)$ & $19(38 \%)$ & 0.294 \\
\hline Symptomatic carotid stenosis & $4(40 \%)$ & $32(64 \%)$ & 0.178 \\
\hline Stroke & $2(20 \%)$ & $21(42 \%)$ & 0.291 \\
\hline Hemispherical TIA & $2(20 \%)$ & $10(20 \%)$ & 1.000 \\
\hline Arterial Hypertension & $10(100 \%)$ & $48(96 \%)$ & 1.000 \\
\hline Hyperlipidemia & $10(100 \%)$ & $36(72 \%)$ & 0.098 \\
\hline Tobacco use & $4(40.0 \%)$ & $13(26 \%)$ & 0.448 \\
\hline Diabetes mellitus & $2(20 \%)$ & $11(22 \%)$ & 1.000 \\
\hline Coronary artery disease & $3(30 \%)$ & $16(32 \%)$ & 1.000 \\
\hline Peripheral occlusive arterial disease & $3(30 \%)$ & $7(14 \%)$ & 0.347 \\
\hline Atrial fibrillation & $1(12.5 \%)$ & $3(6 \%)$ & 0.528 \\
\hline CEA restenosis & $3(30 \%)$ & $6(12 \%)$ & 0.163 \\
\hline Contralateral ICA occlusion & $3(30 \%)$ & $7(14 \%)$ & 0.347 \\
\hline Contralateral ICA stenosis $\geq 70 \%$ & $2(20 \%)$ & $13(26 \%)$ & 1.000 \\
\hline Stenosis $\geq 90 \%$ before CAS & $7(70 \%)$ & $22(44 \%)$ & 0.175 \\
\hline Median follow-up time (month, IQR) & $15(4.7-35.4)$ & $40.2(26.7-59.3)$ & $0.024^{*}$ \\
\hline PSV $>120 \mathrm{~cm} / \mathrm{s}$ after CAS & $4(40 \%)$ & $3(6 \%)$ & 0.012 \\
\hline Re-interventions & $6(60 \%)$ & $0(0 \%)$ & $<0.001$ \\
\hline
\end{tabular}

*significant difference. 
without ISR and a low echogenicity in 5 of patients with ISR (50\%) and in 17 of 50 patients without ISR (34\%). The mean of the reversed echogenicity score was 3,8 in the ISR group and 3,44 in the group without ISR $(\mathrm{p}=0,46)$.

Texture was described as homogenous (score 0 ) or heterogeneous (score 1). In the ISR group 40\% (4/10 patients) scored 0 and $60 \%$ (6/10 patients) scored 1, whereas $46 \%$ (23/50 patients) scored 0 and 54\% (27/50 patients) scored 1 in the control group. Again no significant difference between the two groups was detectable $(p=0,53)$.

The surface was described as either smooth (score 0 ) or irregular (score 1). In the ISR group $10 \%$ (1/10 patients) scored 0 and $90 \%$ (9/10 patients) scored 1, whereas $34 \%$ (17/50 patients) scored 0 and 66\% (33/50 patients) scored 1 in the control group. Again no significant difference between the two groups was detectable $(p=0,62)$.

In the next step, we performed a ROC analysis for TPRS as well as for all individual parameters and calculated sensitivity and specificity, as well as negative predictive value and positive predictive value for each parameter (Table 3). Neither the TPRS score nor one of the individual parameters showed an acceptable validity so that it could be used as a predictive diagnostic test for ISR in patients undergoing CAS.

\section{Discussion}

CAS might be the therapy of choice for patients younger than 70 years [5,9-11]. The on average young age of CAS patients highlights the importance of a good longterm clinical outcome and the need for potentially predictive factors concerning the development of an ISR. Although different approaches, especially regarding laboratory parameters, have been made no reliable parameter is available to date which can sufficiently predict an increased risk of ISR [14,21,26,27].

Duplex sonography (DUS) has been proven over years to be able to assess carotid plaque morphology with regards to plaque surface and the plaque structure. In 2004 Willfort-Ehringer and co-workers applied DUS to assess the influence of pre- interventional plaque morphology on Stent expansion in a 2 years follow up study
[28]. They evaluated the plaque morphology concerning the echoluency of the preinterventional plaque and discriminated between 7 different plaque types ranging from soft to very hard with extensive shadowing. They found that an increased calcification of the pre-interventional plaque composition is associated with a decrease of stent expansion [28] which is known as a risk factor for the development of ISR [12,22].

Bearing these studies in mind we sought to study the influence of pre-interventional plaque morphology on ISR by an easy to use and reliable plaque scoring system.

Intima injury is supposed to be the initial trigger of ISR. Petric et al. showed that the risk of intima injury was lower in calcified plaques in comparison to soft plaques strengthening the hypothesis of the "vulnerable" soft plaque [19]. Therefore, it could be possible that a soft plaque may be associated with a higher risk of the development of an ISR.

We retrospectively analyzed the ultrasound data of patients who underwent CAS in our department between 2003 and 2012 and analyzed the plaque morphology regarding to the TPR score published by Prati et al. [13]. Interestingly we were not able to detect any influence of either scoring parameters on the occurrence of ISR in the course of the evaluation. But, as described by WillfortEhringer and us earlier, we were also able to correlate the echogenicity of the preinterventional plaque with the occurrence of incomplete stenosis dilatation, which is, as we were able to show earlier and again an independent indicator for ISR occurrence [12,28].

One main shortcoming of our study, besides the retrospective design, is the small sample size. We were only able to analyze the data of 10 patients suffering from an ISR and 50 controls, leaving this study underpowered for the detection of small differences within the analyzed factors. However, the results of this study provide evidence that no plaque parameter within the TPRS is able to predict ISR in CAS patients with acceptable validity.

\section{Conclusion}

In the present study we sought to evaluate a standard, easy to use, broadly applicable and reliable system. It

Table 3 Diagnostic validity and statistical data

\begin{tabular}{|c|c|c|c|c|c|}
\hline & TPRS & Inverse TPRS & Echo-genicity & Texture & Surface \\
\hline \multirow[t]{2}{*}{ AUC $(95 \% \mathrm{Cl})$} & 0.54 & 0.64 & 0.46 & 0.53 & 0.62 \\
\hline & $(0.36-0.72)$ & $(0.45-0.83)$ & $(0.25-0.67)$ & $(0.36-0.70)$ & $(0.50-0.74$ \\
\hline Cut-off & $\geq 3$ & $\geq 4$ & $\geq 3$ & $\geq 1$ & $\geq 1$ \\
\hline Sensitivity & 0.70 & 0.80 & 0.20 & 0.60 & 0.90 \\
\hline Specificity & 0.40 & 0.42 & 0.88 & 0.46 & 0.34 \\
\hline $\mathrm{PPV}^{*}$ & 0.06 & 0.08 & 0.09 & 0.06 & 0.07 \\
\hline $\mathrm{PNV}^{*}$ & 0.96 & 0.97 & 0.95 & 0.95 & 0.98 \\
\hline
\end{tabular}

*Positive and negative predictive value given the ISR prevalence of 0.06 in the overall population of CAS-treated individuals of this study. 
might well be, that the non-contrast enhanced DUS of the pre-interventional plaque formation is not sufficient enough and it would be more advisable to investigate a more sophisticated approach either by ultrasound based plaque perfusion imaging, or CT based plaque analysis, lacking the immediate broad applicability. In summary, the pre-interventional assessment of plaque morphology with conventional DUS by using the TPRS is not useful in order to distinguish between patients who are likely to suffer from ISR. Further studies will need to analyze prospectively the usefulness of e.g. Plaque perfusion techniques and DUS or CT techniques in order to reevaluate pre-interventional plaques as possible predictive markers for the development of ISR.

\section{Competing interests}

The authors declare that they have no competing interests.

\section{Authors' contributions}

KW Searched the database, selected the patients, participated in statistical analysis, prepared the manuscript draft and participated in the study design. AK performed the statistical analysis and helped to draft the manuscript. SG participated in creating the database and helped drafting the manuscript. JW participated in creating the database and helped drafting the manuscript. KG participated in creating the database and helped drafting the manuscript. MB participated in study design and outline and helped to draft the manuscript. JL designed the study, participated in drafting the manuscript, did the figure work and analysed the ultrasound data. All authors read and approved the final manuscript.

\section{Author details}

'Department of Neurology, University of Göttingen, Robert-Koch-Str. 40, 37075 Göttingen, Germany. ${ }^{2}$ Department of Psychiatry, University of Mainz, Untere Zahlbacher Str. 8, 55131 Mainz, Germany. ${ }^{3}$ Department of Neurology, University of Mainz, Langenbeckstr. 1, 55131 Mainz, Germany.

Received: 7 August 2013 Accepted: 30 October 2013

Published: 5 November 2013

\section{References}

1. Ringleb PA, Allenberg J, Bruckmann $\mathrm{H}$, et al: $\mathbf{3 0}$ day results from the SPACE trial of stent-protected angioplasty versus carotid endarterectomy in symptomatic patients: a randomised non-inferiority trial. Lancet 2006 , 368(9543):1239-1247.

2. Mas JL, Chatellier G, Beyssen B, et al: Endarterectomy versus stenting in patients with symptomatic severe carotid stenosis. N Engl J Med 2006, 355(16):1660-1671.

3. Economopoulos KP, Sergentanis TN, Tsivgoulis G, Mariolis AD, Stefanadis C: Carotid artery stenting versus carotid endarterectomy: a comprehensive meta-analysis of short-term and long-term outcomes. Stroke 2011, 42(3):687-692.

4. Brott TG, Hobson RW 2nd, Howard G, et al: Stenting versus endarterectomy for treatment of carotid-artery stenosis. N Engl J Med 2010, 363(1):11-23.

5. Bonati LH, Dobson J, Algra A, et al: Short-term outcome after stenting versus endarterectomy for symptomatic carotid stenosis: a preplanned meta-analysis of individual patient data. Lancet 2010, 376(9746):1062-1073.

6. Ederle J, Dobson J, Featherstone RL, et al: Carotid artery stenting compared with endarterectomy in patients with symptomatic carotid stenosis (International Carotid Stenting Study): an interim analysis of a randomised controlled trial. Lancet 2010, 375(9719):985-997.

7. Brott TG, Halperin JL, Abbara S, et al: ASA/ACCF/AHA/AANN/AANS/ACR/ ASNR/CNS/SAIP/SCAI/SIR/SNIS/SVM/SVS guideline on the management of patients with extracranial carotid and vertebral artery disease: executive summary. A report of the American College of Cardiology Foundation/American Heart Association Task Force on Practice Guidelines, and the American Stroke Association, American Association of Neuroscience Nurses, American Association of Neurological Surgeons,
American College of Radiology, American Society of Neuroradiology, Congress of Neurological Surgeons, Society of Atherosclerosis Imaging and Prevention, Society for Cardiovascular Angiography and Interventions, Society of Interventional Radiology, Society of Neurolnterventional Surgery, Society for Vascular Medicine, and Society for Vascular Surgery. Circulation 2011, 124(4):489-532.

8. Carotid Stenting Guidelines Committee: an Inter-collegiate Committee of the RACP (ANZAN, CSANZ), RACS (ANZSVS) and RANZCR: Guidelines for patient selection and performance of carotid artery stenting. Intern Med J 2011, 41(4):344-347.

9. Kastrup A, Gröschel K: Carotid endarterectomy versus carotid stenting: an updated review of randomized trials and subgroup analyses. Acta Chir Belg 2007, 107(2):119-128.

10. Rothwell PM: Poor outcomes after endovascular treatment of symptomatic carotid stenosis: time for a moratorium. Lancet Neurol 2009, 8(10):871-873.

11. Gurm HS, Yadav JS, Fayad P, et al: Long-term results of carotid stenting versus endarterectomy in high-risk patients. N Engl J Med 2008, 358(15):1572-1579.

12. Wasser $\mathrm{K}$, Schnaudigel $\mathrm{S}$, Wohlfahrt J, et al: Clinical impact and predictors of carotid artery in-stent restenosis. J Neurol 2012, 259(9):1896-1902.

13. Prati P, Tosetto A, Casaroli M, et al: Carotid plaque morphology improves stroke risk prediction: usefulness of a new ultrasonographic score. Cerebrovasc Dis 2011, 31(3):300-304.

14. Stojanov D, llic M, Bosnjakovic $P$, et al: New ischemic brain lesions on diffusion-weighted MRI after carotid artery stenting with filter protection: frequency and relationship with plaque morphology. AJNR Am J Neuroradiol 2012, 33(4):708-714.

15. Arnold JA, Modaresi KB, Thomas N, Taylor PR, Padayachee TS: Carotid plaque characterization by duplex scanning: observer error may undermine current clinical trials. Stroke 1999, 30(1):61-65.

16. Kornowski R, Hong MK, Tio FO, Bramwell O, Wu H, Leon MB: In-stent restenosis: contributions of inflammatory responses and arterial injury to neointimal hyperplasia. J Am Coll Cardiol 1998, 31(1):224-230.

17. Farb A, Sangiorgi G, Carter AJ, et al: Pathology of acute and chronic coronary stenting in humans. Circulation 1999, 99(1):44-52.

18. Welt FG, Rogers C: Inflammation and restenosis in the stent era. Arterioscler Thromb Vasc Biol 2002, 22(11):1769-1776.

19. Pericevic I, Lally C, Toner D, Kelly DJ: The influence of plaque composition on underlying arterial wall stress during stent expansion: the case for lesion-specific stents. Med Eng Phys 2009, 31(4):428-433.

20. Group ECSTC: Randomised trial of endarterectomy for recently symptomatic carotid stenosis: final results of the MRC European Carotid Surgery Trial (ECST). Lancet 1998, 351(9113):1379-1387.

21. Wasser K, Schnaudigel S, Wohlfahrt J, Psychogios MN, Knauth M, Gröschel K: Inflammation and in-stent restenosis: the role of serum markers and stent characteristics in carotid artery stenting. PLoS One 2011, 6(7):e22683.

22. Wasser K, Gröschel S, Wohlfahrt J, Gröschel K: Predictors of carotid artery in-stent restenosis. In New trends in Neurosonology and Cerebral Hemodynamics-an update, Volume 1 1-12. Edited by Eva B, Holger P. Munich, Germany: Elsevier publisher; 2012:122-128.

23. Steffen CM, Gray-Weale AC, Byrne KE, Lusby RJ: Carotid artery atheroma: ultrasound appearance in symptomatic and asymptomatic vessels. Aust N Z J Surg 1989, 59(7):529-534.

24. de Bray JM, Baud JM, Delanoy P, et al: Reproducibility in ultrasonic characterization of carotid plaques. Cerebrovasc Dis 1998, 8(5):273-277.

25. de Bray JM, Baud JM, Dauzat M: Consensus Concerning the Morphology and the Risk of Carotid Plaques. Cerebrovasc Dis 1997, 7(5):289-296.

26. Gupta R, Bhatt A, Kassab M, Majid A: Elevated levels of pre-procedural high-sensitivity C-reactive protein is associated with midterm restenosis after extra- and intracranial stenting. J Neuroimaging 2010, 20(1):74-77.

27. Tanemura $\mathrm{H}$, Maeda $\mathrm{M}$, Ichikawa $\mathrm{N}$, et al: High-risk plaque for carotid artery stenting evaluated with 3-dimensional T1-weighted gradient echo sequence. Stroke 2013, 44(1):105-110.

28. Willfort-Ehringer A, Ahmadi R, Gruber D, et al: Arterial remodeling and hemodynamics in carotid stents: a prospective duplex ultrasound study over 2 years. J Vasc Surg 2004, 39(4):728-734.

doi:10.1186/1471-2377-13-163

Cite this article as: Wasser et al:: Plaque morphology detected with Duplex ultrasound before carotid angioplasty and stenting (CAS) is not a predictor of carotid artery in-stent restenosis, a case control study. BMC Neurology 2013 13:163. 